\title{
Potencial hidrogeniônico de dentifrícios e antissépticos com propriedades
}

\section{dessensibilizantes}

\author{
Hydrogenic potential of toothpastes and mouth antiseptics with desensitizing properties \\ Potencial hidrogénico de las pastas dentales y enjuagues bucales con propiedades dessensibilizantes
}

Recebido: 29/11/2021 | Revisado: 06/12/2021 | Aceito: 14/12/2021 | Publicado: 22/12/2021

Luana Laureano Galdino
ORCID: https://orcid.org/0000-0003-3382-3989
Universidade Estadual da Paraíba, Brasil
E-mail: luuanalaureano@ gmail.com
Nívea Maria Nery Gregório
ORCID: https://orcid.org/0000-0002-6310-6756
Universidade Estadual da Paraíba, Brasil
E-mail: nivinhamgn @gmail.com
Ana Cristina Silva Muniz
ORCID: https://orcid.org/0000-0001-5238-9837
Universidade Federal de Campina Grande, Brasil
E-mail: cristinamuniz252@ gmail.com
André Luiz Fiquene de Brito
ORCID: https://orcid.org/0000-0003-4953-8971
Universidade Federal de Campina Grande, Brasil
E-mail: andrefiquene2009@ gmail.com
Helene Soares Moura
ORCID: https://orcid.org/0000-0001-8134-4566
Universidade Estadual da Paraíba, Brasil
E-mail: helenesmoura@ servidor.uepb.edu.br
Nayanna Lana Soares Fernandes
ORCID: https://orcid.org/0000-0003-0453-0001
Universidade Estadual da Paraíba, Brasil
E-mail: fernandesnayanna58@gmail.com
Arella Cristina Muniz Brito
ORCID: https://orcid.org/0000-0001-5482-9592
Universidade Estadual da Paraíba, Brasil
E-mail: arellabrito@gmail.com

\section{Resumo}

Objetivo: Avaliar o pH de três marcas diferentes de dentifrícios e antissépticos utilizados para prevenção e alívio da sensibilidade dentária. Materiais e métodos: Foram utilizadas três marcas diferentes de dentifrícios: Sensodyne PROesmalte ${ }$, Colgate sensitive PRO-alívio $®$, e Colgate Total 12®, todas disponíveis comercialmente. Para análise dos dados, pesou-se $5 \mathrm{~g}$ de cada marca em balança analítica de precisão em seguida adicionou-se $15 \mathrm{ml}$ de água destilada para leitura posterior. Enquanto, os antissépticos considerados para a pesquisa foram Elmex sensitive ${ }^{\circledR}$, Sensodyne ${ }^{\circledR}$ e Colgate Total 12®. Utilizou-se 40mL de cada marca. O potencial hidrogeniônico $(\mathrm{pH})$ foi mensurado pelo método potencio métrico em pHmetro digital. Os testes foram realizados em quintuplicata e os dados analisados por ANOVA/one-way com nível de significância de 5\%. Resultados: Os valores de pH dos dentríficios variaram entre

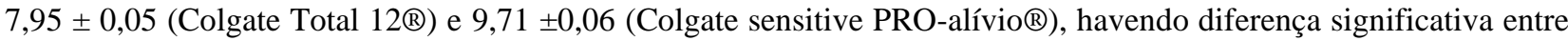
todas as marcas avaliadas $(p<0,05)$. Em contrapartida, as médias do $\mathrm{pH}$ dos antissépticos bucais obtidas de cada

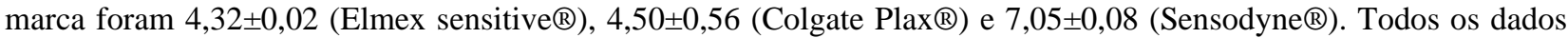
foram analisados estatisticamente e o resultado mostrou que as duas primeiras marcas não apresentaram um $\mathrm{pH}$ ácido e não diferiram entre si $(p>0,05)$. Mas a Sensodyne ${ }^{\circledR}$ apresentou um $\mathrm{pH}$ mais elevado e neutro e com diferenças significativas quando comparado as duas marcas anteriores $(p<0,05)$. Conclusão: Os dentifrícios encontram-se na faixa de $\mathrm{pH}$ ideal para o uso e os antissépticos bucais não apresentam $\mathrm{pH}$ potencialmente erosivo, pois encontram-se dentro da faixa de segurança.

Palavras-chave: Sensibilidade da dentina; Dentifrícios; Antissépticos bucais.

\begin{abstract}
Objective: To evaluate the $\mathrm{pH}$ of three different brands of toothpaste and antiseptic used to prevent and relieve tooth sensitivity. Materials and methods: Three different brands of dentifrices were used: Sensodyne PRO-enamel@, Colgate sensitive PRO-alívioß, and Colgate Total $12 \AA$, all commercially available. For data analysis, $5 \mathrm{~g}$ of each brand was weighed on a precision analytical balance, then $15 \mathrm{ml}$ of distilled water were added for later reading.
\end{abstract}


Meanwhile, the antiseptics considered for the research were Elmex sensitive $₫$, Sensodyne® and Colgate Total $12 ®$. $40 \mathrm{ml}$ of each brand was used. The hydrogen ion potential $(\mathrm{pH})$ was measured by the metric potential method in a digital $\mathrm{pH}$ meter. The tests were performed in quintuplicate and the data analyzed by ANOVA/one-way with a significance level of 5\%. Results: The dentifrice $\mathrm{pH}$ values ranged between $7.95 \pm 0.05$ (Colgate Total 12®) and 9.71 \pm 0.06 (Colgate sensitive PRO-alívio®), with a significant difference between all brands evaluated $(\mathrm{p}<0.05)$. In

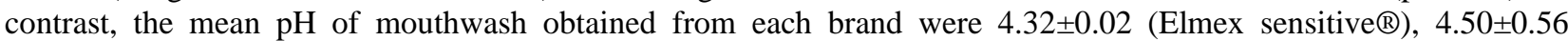
$\left(\right.$ Colgate Plax ${ }^{\circledR}$ ) and 7.05 \pm 0.08 (Sensodyne ${ }^{\circledR}$ ). All data were statistically analyzed and the result showed that the first two brands did not present an acidic $\mathrm{pH}$ and did not differ from each other $(\mathrm{p}>0.05)$. But Sensodyne® had a higher and neutral $\mathrm{pH}$ and with a significant difference when compared to the two previous brands $(\mathrm{p}<0.05)$. Conclusion: Toothpastes are in the ideal $\mathrm{pH}$ range for use and mouthwashes do not have a potentially erosive $\mathrm{pH}$, as they are within the safety range.

Keywords: Dentin sensitivity; Toothpaste; Mouth antiseptics.

\section{Resumen}

Objetivo: Evaluar el pH de tres marcas diferentes de pasta de dientes y antisépticos utilizados para prevenir y aliviar la sensibilidad dental. Materiales y métodos: Se utilizaron tres marcas diferentes de dentífricos: Sensodyne PROenamel®, Colgate Sensitive PRO-alívio® y Colgate Total 12®, todos disponibles comercialmente. Para el análisis de los datos, se pesaron $5 \mathrm{~g}$ de cada marca en una balanza analítica de precisión, luego se agregaron $15 \mathrm{ml}$ de agua destilada para su posterior lectura. Mientras tanto, los antisépticos considerados para la investigación fueron Elmex sensitivo ${ }^{\circledR}$, Sensodyne ${ }^{\circledR}$ y Colgate Total $12 ®$. Se utilizaron $40 \mathrm{ml}$ de cada marca. El potencial de iones de hidrógeno $(\mathrm{pH})$ se midió mediante el método de potencial métrico en un medidor de $\mathrm{pH}$ digital. Las pruebas se realizaron por quintuplicado y los datos se analizaron mediante ANOVA / one-way con un nivel de significancia del $5 \%$. Resultados: Los valores de pH del dentífrico oscilaron entre 7,95 \pm 0,05 (Colgate Total 12®) y 9,71 \pm 0,06 (Colgate Sensible PRO-alívio®), con diferencia significativa entre todas las marcas evaluadas ( $\mathrm{p}<0,05)$. En contraste, el $\mathrm{pH}$ medio de los enjuagues bucales obtenidos de cada marca fue de 4,32 $\pm 0,02$ (Elmex sensitivo®), 4,50 $\pm 0,56$ (Colgate Plax $\left.{ }^{\circledR}\right)$ y 7,05 $\pm 0,08$ (Sensodyne () ). Todos los datos fueron analizados estadísticamente y el resultado mostró que las dos primeras marcas no presentaron un $\mathrm{pH}$ ácido y no difirieron entre sí ( $\mathrm{p}>0.05$ ). Pero Sensodyne ${ }^{\circledR}$ tuvo un $\mathrm{pH}$ más alto y neutro y con una diferencia significativa en comparación con las dos marcas anteriores ( $\mathrm{p}<0.05)$. Conclusión: Las pastas de dientes están en el rango de $\mathrm{pH}$ ideal para su uso y los enjuagues bucales no tienen un $\mathrm{pH}$ potencialmente erosivo, ya que están dentro del rango de seguridad.

Palabras clave: Sensibilidad dentinaria; Pasta dental; Antisépticos bucales.

\section{Introdução}

A hipersensibilidade dentinária é uma condição bucal dolorosa comum entre pacientes, sendo considerada um problema multifatorial, mas a erosão dentária e a retração gengival são alguns dos principais fatores dessa alteração (Douglasde-oliveira, Paiva \& Cota, 2017). Comumente é relacionada à exposição de dentina e à perda da estrutura do esmalte frente a uma agressão biológica, física ou química (Amaral, Galafassi, \& Butzi, 2019).

É caracterizada como uma sensibilidade exagerada em resposta a estímulos químicos, táteis, térmicos ou osmóticos impactando de forma negativa a qualidade de vida do indivíduo (Molina et al., 2017). A etiologia ainda não foi completamente compreendida, porém, a Teoria Hidrodinâmica, proposta por Brännström na década de 1960 é a mais aceita que estabelece que o movimento dos fluidos nos canalículos dentinário resulta na dor dentinária (Sharda et al., 2018).

Alternativas como medidas preventivas e tratamentos se tornam essenciais para amenizar os efeitos da hipersensibilidade, assim como controlar o desconforto para os pacientes. Os métodos utilizados para o este controle estão geralmente relacionados às mudanças na alimentação, controle da força aplicada na higiene oral e utilização de produtos específicos para diminuição da dor (Gaona, 2018). Dentre as opções de produtos específicos, tem sido recomendada a utilização de produtos com princípios ativos que possam auxiliar na diminuição da sensibilidade (Molina et al., 2017).

O tratamento da hipersensibilidade engloba diferentes técnicas podendo ser realizadas no consultório com a aplicação de dessensibilizantes tópicos, restauração da dentina exposta ou até mesmo com recobrimento radicular dependendo da extenseão do caso, no entanto pode haver também o controle diário por parte do paciente, e umas dessas alternativas é por meio da utilização de dentifrícios e antissépticos específicos para esta demanda, por serem menos invasivos e acessíveis financeiramente (Brignardello-Petersen, 2019; Oliveira et al., 2021). 
Os dentifrícios comercializados com o intuito de diminuir a sensibilidade, contêm componentes que auxiliam no aumento da resistência ao desgaste e, dentre estes, encontram-se Fosfossilicato de Cálcio e Sódio, Silicato de Cálcio, Fosfato de Sódio, Arginina e Carbonato de Cálcio. Apresentam como vantagem um tratamento menos invasivo, e mais aplicável para os indivíduos, uma vez que financeiramente é mais viável (Gaona, 2018; Elgamily et al., 2019; Vilhena et al., 2020). Os hábitos que o paciente possui que colaboram para a sintomatologia, assim como seu histórico com a hipersensibilidade, são formas para se chegar a um planejamento adequado (Regiani, Rocha, Tognetti, \& Andrade, 2021). Os dentifrícios como método de tratamento paliativo, devem estar dentro de uma faixa de segurança entre 4,5 a 10,5, de acordo com o Padrão Internacional utilizado pelo Brasil Instituto de Metrologia para que se tornem ideal para o uso, de modo que traga contribuições satisfatórias (Brito et.al, 2015).

Em relação aos antissépticos bucais, os mesmos podem auxiliar na prevenção e no tratamento da sensibilidade da dentina, uma vez que apresentem em sua composição nitrato de potássio, fluoreto de sódio, citrato de potássio, fluoreto de sódio ou uma mistura de fluoretos juntamente com um pH seguro (Molina et al., 2017; Hall et al., 2019). Além disso, a literatura tem evidenciado que o uso doméstico dos antissépticos bucais apresentam bons resultados na redução de escores utilizados para mensurar a sensação dolorosa relacionada à sensibilidade (Molina et al.2017). A correta indicação e orientação adequada por um cirurgião dentista pode apresentar um resultado satisfatório em pacientes acometidos por hipersensibilidade dentária (Brignardello-Petersen, 2019; Sharda et al., 2018).

O mecanismo de ação dos produtos envolvidos na prevenção e/ou tratamento da hipersensibilidade dentinária está relacionado à dessensibilizar os nervos expostos ou ocluir os túbulos dentinários. Diante desse contexto, as formulações modernas de dentifrícios e antissépticos precisam liberar seus componentes de forma controlada e apresentar propriedades físico-químicas satisfatórias para atender a este objetivo. Dentre essas propriedades, uma que se destaca é o potencial hidrogênionico $(\mathrm{pH})$ de uma solução, que atua como parâmetro para especificar a acidez ou basicidade de uma solução. O pH é considerado um fator fundamental na avaliação do potencial erosivo de bebidas e alimentos, como também de produtos de higiene oral, funcionando como um auxiliar na diminuição da abrasividade da formulação e conseqüentemente prevenindo e/ou diminuindo a hipersesnibilidade (Meira et al., 2020; de Oliveira et al., 2017; Magalhães, 2017; Carvalho 2015).

Dessa maneira, o objetivo do presente estudo foi avaliar o pH de três marcas diferentes de dentifrícios e antissépticos utilizados para prevenção e alívio da hipersensibilidade dentária. A hipótese nula testada foi que tanto os dentifrícios quanto os antissépticos não apresentam diferenças significativas relacionadas ao $\mathrm{pH}$.

\section{Metodologia}

\section{Desenho experimental}

Foi realizado um estudo in vitro em que foi avaliado o potencial hidrogeniônico $(\mathrm{pH})$ de diferentes marcas de produtos de autoconsumo utilizados para redução de sensibilidade dentária. Os produtos de autoconsumo foram divididos em dois grupos para análises referentes a dentifrícios e antissépticos bucais.

Foram selecionados produtos disponíveis comercialmente em farmácias e supermercados brasileiros que apresentavam a propriedade de combater a hiperssensibilidade. O tamanho da amostra considerou um total de 3 marcas de dentifrícios e e 3 marcas diferentes de antissépticos, totalizando um tamanho amostral de 3 para cada grupo $(n=3)$. As marcas foram selecionadas ao acaso de acordo com a disponibilidade. As composições químicas dos dentifrícios (Quadro 1) e dos antissépticos (Quadro 2) estão descritas a seguir. O fator em estudo foi a diferença entre as marcas disponíveis.

Todas as análises foram realizadas em quintuplicata e os equipamentos utilizados foram previamente calibrados de acordo com suas especificações dos fabricantes. Os dados foram coletados no Laboratório de Gestão e Tratamento de Resíduos (LABGER), localizado na Universidade Federal de Campina Grande, Paraíba, Brasil. 


\section{Avaliação da composição química}

Em relação a composição química dos dentifrícios assim como dos enxaguantes utilizados, a mesmas foram obtidas a partir da leitura dos ingredientes disponibilizada nas embalagens de cada produto. Esta etapa foi realizada para identificação dos ingredientes ativos e quais seriam suas possíveis interferências nos resultados do potencial hidrogeniônico ( $\mathrm{pH}$ ) obtidos.

\section{Avaliação do pH dos dentifrícios}

Para a mensuração do $\mathrm{pH}$ dos dentifrícios avaliados, foram pesados $5 \mathrm{~g}$ de cada em uma balação de precisão (BEL Engineering ${ }^{\circledR}$, Piracicaba, Brasil). Em seguida, para facilitar a leitura da amostra, foi realizada a diluição com $15 \mathrm{~mL}$ de água destilada com auxílio de um agitador termomagnético à temperatura ambiente de $25^{\circ} \mathrm{C}$ (Biomixer®, Ribeirão Preto, Brasil). Os valores de pH foram mensurados utilizando um pHmetro digital (Digimed®, São Paulo, Brasil) pré-calibrado em soluções de 4 e 7.

\section{Avaliação do pH dos antissépticos}

O volume de $40 \mathrm{~mL}$ de cada marca avaliada foi obtido por meio da mensuração em uma proveta de precisão. Em seguida o volume de cada antisséptico mensurado foi transferido para seu respectivo Becker de análise. A leitura dos valores de pH endógenos também foram mensurados utilizando um pHmetro digital (Digimed®, São Paulo, Brasil) pré-calibrado em soluções de 4 e 7.

\section{Análise estatística}

O desenho experimental do presente estudo propôs a utilização de um fator de análise, a marca de cada produto. Desse modo, após aplicação de teste de normalidade Shapiro-Wilk por grupo e de homocedasticidade de Levene, foi observado que os dados apresentaram distribuição do tipo normal. Nesse contexto, o teste escolhido foi a análise de variância ANOVA (oneway) utilizando o software estatístico Minitab®19 (Minitab Inc., State College, PA, USA) (2014). Para identificação das diferenças entre as marcas, foi aplicado o Teste de Tukey. Todas as análises com foram atribuídas uma significância de 5\%.

\section{Resultados}

\section{Avaliação do $\mathrm{pH}$ dos dentifrícios}

Os valores de pH variaram entre 7,95 $\pm \mathbf{0 , 0 5}$ (Colgate Total 12®) e 9,71 $\pm \mathbf{0 , 0 6}$ (Colgate sensitive PRO-alívio®). Tais resultados indicam que todas as marcas apresentaram um pH alcalino. Além disso, houve diferença significativa entre todas as marcas avaliadas $(\mathrm{p}<0,05)$.

\section{Avaliação do pH dos antissépticos}

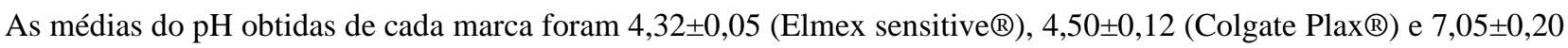
(Sensodyne $\left.{ }^{\circledR}\right)$. As duas primeiras marcas apresentaram um $\mathrm{pH}$ ácido e não diferiram entre si $(\mathrm{p}>0,05)$. O enxaguante Sensodyne ${ }^{\circledR}$ apresentou um pH mais elevado e neutro e com diferença significativas quando comparado as duas marcas anteriores $(\mathrm{p}<0,05)$.

\section{Discussão}

Os dentifrícios e antissépticos, mostram-se um dos meios mais comuns para ação dessensibilizante, uma vez que se tornam produtos farmacêuticos sofisticados no mercado atual, podendo garantir eficácia no tratamento da hipersensibilidade dentinária (HD) (Paz et al., 2021). 
Dessa maneira, os valores de $\mathrm{pH}$ referente ao estudo dos dentifrícios variaram entre 7,95 $\pm 0,05$ e 9,71 $\pm 0,06$, o que indicou um $\mathrm{pH}$ alcalino, dentro da margem de segurança, ideal para o uso, de modo que contribui para o alívio da dor pela hipersensibilidade por ocasionar menor desgaste, e serem menos erosivos. Além disso, houve diferença significativa entre todas as marcas avaliadas $(p<0,05)$. Nesse contexto, está de acordo com estudos previamente realizados, pois o ideal dos valores de $\mathrm{pH}$ dentifrícios se enquadra na faixa equivalente a valores alcalinos (Cavalcanti, Cardoso \& Brito, 2015). Além disso valores que apresentam um potencial mais ácido, podem ocasionar uma possível ação erosiva, pois os que apresentam perfil ácido não seriam interessante para indivíduos que sofrem com a hipersensibilidade, pois haveria um maior desgaste do esmalte. (Cavalcanti, Cardoso \& Brito, 2015).

Além disso, dentifrícios com um baixo grau de abrasividade, e mais alcalinos, se tornam o mais ideal para a hipersensibilidade dentinária, e isso dependerá também do diagnóstico e histórico do paciente. Dentifrícios com componentes como fosfosilicato de cálcio e silicato de cálcio, foram considerados importantes para um maior fechamento dos túbulos dentinários, favorecendo o tratamento. (Gaona, 2018).

As evidências científicas relatam que dentifrícios à base de Arginina como grandes contribuintes na redução da hipersensibilidade dentinária, o que pode ser observado no creme dental Colgate Sensitive PRO-alívio® (Cury \& Tenuta, 2014). Assim como dentifrícios que apresentam nitrato de potássio em sua composição, pois apresentam alta concentração de flúor, garantem a proteção contra a erosão dentária e, consequentemente, baixa abrasividade, tendo efeito satisfatório na hipersensibilidade dentinária, atuando na dessensibilização dos nervos pulpares (João-Souza et.al, 2019). Somado a isto, dentifrícios compostos por quitosana e íons estanosos, obtiveram precipitados os quais protegeram a dentina contra desafios ácidos, os quais resultam em uma camada com menor abrasividade na superfície (João-Souza et.al, 2019).

Em relação a prescrição de antissépticos bucais como adjuvante à escovação dentária também pode ser útil para reduzir a condição dolorosa relatada pelo paciente, principalmente os que contêm nitrato de potássio, fluoreto de sódio, citrato de potássio ou uma mistura de fluoretos (Molina et al., 2017; Hall et al., 2019). Dentre as marcas avaliadas na presente pesquisa, a Sensodyne® é a única que apresenta nitrato de potássio na sua composição e Colgate Plax® fluoreto de sódio, enquanto a Elmex sensitive ${ }^{\circledR}$ possui mistura de fluoretos.

Os resultados de $\mathrm{pH}$ avaliado dos antissépticos bucais do presente estudo mostrou diferença significativas entre Sensodyne ${ }^{\circledR}$ quando comparado com as marcas Elmex sensitive ${ }^{\circledR}$ e Colgate Plax ${ }^{\circledR}(p<0,05)$. No entanto, de acordo com os testes avaliados os três antissépticos bucais não apresentam $\mathrm{pH}$ potencialmente erosivo, pois se encontram dentro da faixa de segurança. Mas, a Sensodyne ${ }^{\circledR}$ foi considerada a melhor opção por apresentar um pH mais elevado e neutro.

As evidências científicas sobre antissépticos bucais que auxiliem no alívio da hipersensibilidade dentária são bastante escassas, não possuindo muitos estudos para fins comparativos adequados sobre diferentes princípios ativos (BrignardelloPetersen, 2019; Sharda et al., 2018). Embora possua revisão sistemática e estudos de coorte que apontam para efeitos positivos sobre os sintomas dos pacientes com uso desse produto como adjuvante na higiene bucal, existe ainda assim uma limitação de estudos na literatura (Gaona, 2018).

A utilização de dentifrícios, assim como a forma e o tempo de escovação, é algo que que também deve ser discutido, uma vez que a mesma pode trazer benefícios ou malefícios, já que cerdas duras, assim como técnica de escovação errada, também pode interferir na hipersensibilidade do paciente. Contudo, sendo o dentifrício de menor abrasividade, garante um desgaste não irreversível (Gaona, 2018). As limitações quanto ao nível de abrasividade dos dentifrícios com relação à dentina (RDA) são notórias, uma vez que nas embalagens não há informações para facilitar e orientar a escolha do creme dental, o que poderia ser considerado essencial pelos fabricantes (Rios et.al, 2014).

Dessa forma, é perceptível uma restrição de estudos voltados para a prevenção, alívio e tratamento da hipersensibilidade dentinária com o uso de dentifrícios e antissépticos bucais. Em vista disso, são necessários mais estudos 
como ensaios clínicos longitudinais bem delineados e revisões sistemáticas para fortalecer o embasamento científico e o uso doméstico dos dentifrícios e antissépticos bucais dessensibilizantes com diferentes outros agentes ativos, uma vez que eles apresentam resultados bastante positivos em relação a sensibilidade e um bom custo benefício.

\section{Considerações Finais}

Os dentifrícios e testados encontram-se na faixa de $\mathrm{pH}$ de segurança para o uso, o que é desejável para o contato com os dentes e cavidade oral. Contudo, os pH mesmo dentro da faixa de segurança diferenciaram em relação aos valores e a composição dos materiais de acordo com a marca escolhida. Dessa forma, a Colgate sensitive PRO-alívio® como creme dental e a Sensodyne $®$ como enxaguatório bucal foram consideradas as melhores opções por apresentar um pH mais elevado.

\section{Referências}

Brignardello-Petersen, R. (2019). Using potassium oxalate mouthrinse for 4 weeks is probably more effective than using a placebo to treat dentin hypersensitivity. The Journal Of The American Dental Association, 150 (2), 9-11.

Brito, A. C. M.; Dantas, L. R.; De Brito, A. L.; Muniz, A. C.; Ramos, I. A.; Cardoso, A. M.; Xavier, A. F. e Cavalcanti, A. L. (2015). Loss on drying, calcium concentration and $\mathrm{pH}$ of fluoride dentifrices. Contemporary clinical dentistry, 6 (1), 72-76.

Cury, J. A. e Tenuta, L. M. A. (2014). Evidence-based recommendation on toothpaste use. Brazilian Oral Research, 28 (1), 1-7.

Douglas-de-Oliveira, D. W.; Paiva, S. M. e Cota, L. O. M. (2017). Etiologia, epidemiologia e tratamento da hipersensibilidade dentinária: uma revisão de literatura. Revista Perio, 27(1), 76-85.

Oliveira, A. F. B.; Sampaio, F. C.; Meira, I. A.; Bezerra, M. G. P. G.; Fernandes, N. L. S.; Paiva, V. M. S. (2021). Erosive Potential of Industrialized Teas: An in vitro study. Pesquisa Brasileira em Odontopediatria e Clínica Integrada., v.17, p.1 - 7, 2017. J Clin Exp Dent, 13(1), 48-55.

Elgamily, H.; Safwat, E.; Soliman. Z.; Salama, H.; El-Sayed, H. e Anwar, M. (2019). Antibacterial and remineralization efficacy of casein phosphopeptide, glycomacropeptide nanocomplex, and probiotics in experimental toothpastes: an in vitro comparative study. Eur J Dent, 13, $391-398$.

Gaona, R. M. L. (2019). Hipersensibilidade dentinária e desgaste dental: abordagem epidemiológica, sobre auto-percepção e conhecimento, caracterização física e biológica e estudo in situ do desgaste e oclusão tubular de cremes dentais dessensibilizantes e/ou anti-erosivos (TCC). Universidade de São Paulo.

Hall, C.; Sufi, F. e Constantin, P. (2017). Efficacy of an experimental $3 \%$ potassium nitrate mouthwash in providing long-term relief from dentin hypersensitivity: An 8-week randomized controlled study (Study 2). Am J Dent, 30 (6), 335-342.

Hall, C.; Sufi, F.; Milleman, J. L. e Milleman, K. R. (2019). Efficacy of a 3\% potassium nitrate mouthrinse for the relief of dentinal hypersensitivity. The Journal Of The American Dental Association, 150 (3), 204-212.

Hines, D.; Xu, S.; Stranick, M.; Lavender, S.; Pilch, S.; Zhang, Y. P.; Sullivan, R.; Montesani, L. Montesani, L. e Mateo, Luis, R. (2019) Effect of a stannous fluoride toothpaste on dentinal hypersensitivity. The Journal Of The American Dental Association, 150 (4), 47-59.

Hu, M. L.; Zheng, G.; Zhang, Y. D.; Yan, X; Li, X. C.; Lin, H. (2018). Effect of desensitizing toothpastes on dentine hypersensitivity: a systematic review and meta-analysis. Journal Of Dentistry, 75 (2), 12-2.

João-Souza, S. H.; Scaramucci, I. T.; Borges, A. B.; Lussi, A.; Carvalho, T. S. e Aranha, A. C. C. (2019). Influence of desensitizing and anti-erosive toothpastes on dentine permeability: an in vitro study. Journal Of Dentistry, 89 (2), 103-176.

Krikheli, N. I.; Pustovoit, E. V. e Suvkova, E. I. (2019). The effectiveness of Sensodyne Instant effect toothpaste for dentin hypersensitivity treatment. Stomatologiya, 98 (6), 22.

Lotti Amaral, M.; Galafassi, D. e Butze, J. (2019). Avaliação de dois diferentes agentes dessensibilizantes no tratamento da hipersensibilidade dentinária: Relato de caso. Journal of Oral Investigations, 8(2), 84-100.

Magalhães, A. C.; Oliveira, R. C. D. E.; Buzalaf, M. A. R. (2017). Bioquímica básica e bucal. Editora: Guanabara Koogan, Rio de Janeiro.

Martins, C. C.; Firmino, R. T.; Riva, J. J.; Ge, L.; Carrasco-labra, A.; Brignardello-Petersen, R.; Colunga-Lozano, L. E.; Granville-Garcia, A. F.; Costa, F. O. e Yepes-Nuñez, J. J. Desensitizing Toothpastes for Dentin Hypersensitivity: a network meta-analysis. Journal Of Dental Research, 99 (5), $514-522$.

Mounika, A; Mandava, J; Roopesh, B. e Karri, G. (2018). Clinical evaluation of color change and tooth sensitivity with in-office and home bleaching treatments. Indian Journal Of Dental Research, 29(4), 423.

Molina, A.; García-Gargallo, M.; Montero, E.; Tobías, A.; Sanz, M. e Martín, C (2017). Clinical efficacy of desensitizing mouthwashes for the control of dentin hypersensitivity and root sensitivity: a systematic review and meta-analysis. Int J Dent Hyg, 15(2), 84-94.

Oliveira, E. C.; Viana, A. R.; Serafin, M. B.; Pompeu, L. D.; Manfron, M. P. (2021). Antimicrobial activity of Glechon spathulata BENTH crude extract and fractions. Research, Society and Development, 10 (16), 247-251. 
Research, Society and Development, v. 10, n. 17, e111101724046, 2021

(CC BY 4.0) | ISSN 2525-3409 | DOI: http://dx.doi.org/10.33448/rsd-v10i17.24046

Regiani, B. C.; Rocha, H. N.; Tognetti, V. M. e Andrade, A. P. (2020). Hipersensibilidade dentinária em lesões cervicais não cariosas: etiologia e tratamento. Archives

Rios, A. C. F.; Lopes, S. C. F. L.; Dantas, T. S., Oliveira, V. M. B. e Santos, L. B. (2014). Abrasivos: Uma análise de dentifrícios comercializados em Salvador. Revista Bahiana de Odontologia., 5(3): 141-152.

Sharda, S.; Prasad, K. V. V.; Shetty, P. J. e Nikhil, K. (2018). Effectiveness of Desensitizing Dentifrice and Mouthwash on Dentin Hypersensitivity and Tooth Remineralization. Contemp Clin Dent, 9(3), 415-420.

Seong, J.; Newcombe, R. G.; Matheson, J. R.; Weddell, L.; Edwards, M. e West, N. X. (2020). A randomised controlled trial investigating efficacy of a novel toothpaste containing calcium silicate and sodium phosphate in dentine hypersensitivity pain reduction compared to a fluoride control toothpaste. Journal Of Dentistry, 98 (3), 103-120.

West, N. X..; He, T.; Zou, Y.; Digennaro, J.; Biesbrock, A. e Davies, M. (2021). Bioavailable gluconate chelated stannous fluoride toothpaste meta-analyses: effects on dentine hypersensitivity and enamel erosion. Journal Of Dentistry, 105 (2), 103-116.

Vilhena, F. V.; Polassi, M. R.; Paloco, E. A. C.; Alonso, R. C.; Guiraldo, R. D. e D'Alpino, P. H. (2020). Effectiveness of toothpaste containing REFIX technology against dentin hypersensitivity: a randomized clinical study. J Contemp Dent Pract, 21 (2), 609-614. 